\title{
ANXA2 Gene
}

National Cancer Institute

\section{Source}

National Cancer Institute. ANXA2 Gene. NCI Thesaurus. Code C105376.

This gene is involved in skeletal system development. 